\title{
RESEARCH
}

Open Access

\section{What do we need to know? Data sources to support evidence-based decisions using health technology assessment in Ghana}

Samantha A. Hollingworth ${ }^{1 *} \mathbb{D}$, Laura Downey ${ }^{2}$ D, Francis J. Ruiz ${ }^{3} \mathbb{D}$, Emmanuel Odame $^{4} \mathbb{D}$, Lydia Dsane-Selby ${ }^{5} \mathbb{D}$, Martha Gyansa-Lutterodt ${ }^{6}$ (D) Justice Nonvignon ${ }^{7}$ (D) and Kalipso Chalkidou ${ }^{3,8}$ (D)

\begin{abstract}
Background: Evidence-based decision-making for prioritising health is assisted by health technology assessment $(\mathrm{HTA})$ to integrate data on effectiveness, costs and equity to support transparent decisions. Ghana is moving towards universal health coverage, facilitated mainly by the National Health Insurance Scheme (NHIS) established in 2003. The Government of Ghana is committed to institutionalising HTA for priority-setting. We aimed to identify and describe the sources of accessible data to support HTA in Ghana.

Methods: We identified and described data sources encompassing six main domains using an existing framework. The domains were epidemiology, clinical efficacy, costs, health service use and consumption, quality of life, and equity. We used existing knowledge, views of stakeholders, and searches of the literature and internet.

Results: The data sources for each of the six domains vary in extent and quality. Ghana has several large data sources to support HTA (e.g. Demographic Health Surveys) that have rigorous quality assurance processes. Few accessible data sources were available for costs and resource utilisation. The NHIS is a potentially rich source of data on resource use and costs but there are some limits on access. There are some data on equity but data on quality of life are limited.

Conclusions: A small number of quality data sources are available in Ghana but there are some gaps with respect to HTA based on greater use of local and contextualised information. Although more data are becoming available for monitoring, challenges remain in terms of their usefulness for HTA, and some information may not be available in disaggregated form to enable specific analyses. We support recent initiatives for the routine collection of comprehensive and reliable data that is easily accessible for HTA users. A commitment to HTA will require concerted efforts to leverage existing data sources, for example, from the NHIS, and develop and maintain new data (e.g. local health utility estimates). It will be critical that an overarching strategic and mandatory approach to the collection and use of health information is developed for Ghana in parallel to, and informed by, the development of HTA approaches to support resource allocation decisions. The key to HTA is to use the best available data while being open about its limitations and the impact on uncertainty.
\end{abstract}

Keywords: Health technology assessment, Ghana, data, health information systems

\footnotetext{
* Correspondence: s.hollingworth@uq.edu.au

${ }^{1}$ School of Pharmacy, University of Queensland, 20 Cornwall St, Woolloongabba, QLD 4102, Australia

Full list of author information is available at the end of the article
}

C C The Author(s). 2020 Open Access This article is licensed under a Creative Commons Attribution 4.0 International License, which permits use, sharing, adaptation, distribution and reproduction in any medium or format, as long as you give appropriate credit to the original author(s) and the source, provide a link to the Creative Commons licence, and indicate if changes were made. The images or other third party material in this article are included in the article's Creative Commons licence, unless indicated otherwise in a credit line to the material. If material is not included in the article's Creative Commons licence and your intended use is not permitted by statutory regulation or exceeds the permitted use, you will need to obtain permission directly from the copyright holder. To view a copy of this licence, visit http://creativecommons.org/licenses/by/4.0/. The Creative Commons Public Domain Dedication waiver (http://creativecommons.org/publicdomain/zero/1.0/) applies to the data made available in this article, unless otherwise stated in a credit line to the data. 


\section{Background}

Every health system is challenged when balancing a finite budget with increasing demands on health resources. Setting effective priorities that maximise health gains necessarily requires difficult trade-offs to be made. These challenges are particularly acute in low- and middleincome countries (LMICs). Irrespective of jurisdiction, understanding trade-offs is arguably necessary to help ensure value for money and that waste is minimised $[1,2]$. When setting health priorities, decisions can be better informed through the use of credible and fit-for-purpose evidence on where and how money is being spent in the health system, outcomes of interaction with the health service, and the general 'healthiness' of the population. Such information can be synthesised in order to understand value-formoney of current and future investments in the health system and the impact on health through increasing efficiency. Health technology assessment (HTA) represents a commonly used approach to synthesising evidence on the effectiveness and costs of an intervention, which also aims to consider social value judgements as part of a process to inform efficient and equitable resource allocation [1, 2]. While the specific methods and processes vary according to context, HTA informs health spending decisions in almost all high-income countries [3], and increasingly in middle-income countries such as Thailand $[4,5]$ and South Africa [6, 7], and regions including the Middle East and North Africa $[8,9]$. The World Health Assembly Resolution of 2014 on Health Intervention and Technology Assessment recognizes the importance of this approach as a key element of any strategy for achieving universal health coverage (UHC) $[2,10]$.

The Government of Ghana has enacted legislation and earmarked funds for UHC. The National Health Insurance Scheme (NHIS), established in 2003 [11], offers a comprehensive healthcare package to all residents of Ghana but coverage is only for $38 \%$ of the population [12]. Almost half (46\%) of NHIS expenditure is spent on medicines [13], where the two largest medicine classes antibiotics (29\%) and antihypertensives (14\%) - account for most of the medicines expenditure [13]. In this context, an HTA pilot study was initiated by the Ghanaian Ministry of Health (MoH) in 2016 to examine the costeffectiveness of antihypertensives in the NHIS [14]. This pilot catalysed a partnership between government and academia, demonstrating the importance of institutional collaboration in evidence-informed processes, and how HTA can inform NHIS spending decisions to improve allocative efficiency [15]. In the national medicines policy [16], the Government has outlined its vision to institutionalise HTA as part of its plan towards achieving UHC and has explicitly committed to using HTA evidence to inform prioritisation for the essential medicines list [17] and standard treatment guidelines [18].
Evidence-based policy-making depends on the availability of reliable, locally generated evidence. High-quality and timely health data creates a strong foundation for a highfunctioning health system [3], but there is a marked absence of reliable health information systems in LMICs. In an important first step towards recognising and addressing shortcomings in its health data ecosystem, the Government held a forum in 2017, the Data for Sustainable Development roadmap, and committed to increasing the emphasis on enhancing routine administrative data and integrating new types of data into official statistics $[2,19,20]$. Although there is not a comprehensive suite of robust local evidence, Ghanaian policy-makers can continue to engage in evidence-informed policy-making noting the challenges for the effective implementation of HTA [2, 20]. Taking stock of the information available within the Ghanaian health system is an essential first step towards planning for the routine generation and use of HTA into the evidence-based priority-setting process in Ghana. We aimed to describe the publicly available data sources in Ghana that can support HTA and evidence-based priority-setting.

\section{Methods}

We identified the information required to support HTA based on previous work from our group [21]. We focused on six 'data' domains, namely epidemiology, clinical efficacy, costs, health service use, quality of life and equity [21]. We identified data sources based on our local experience, using the internet to search the literature, Ghana government and other websites (e.g. non-government organisations), and liaising with key informants for additional input.

\section{Results}

These six data source domains encompass many of the key elements needed for a well-conducted economic evaluation, with context-relevant details specified ex ante based on a suitable reference case [21]. iDSI developed a reference case for adaptation to local situations [22] and it provides a systematic way to inform the conduct and reporting of economic evaluations. We have summarised the main data sources available in Ghana (Table 1) and those used for the Ghana hypertension economic evaluation (Table 2) [14].

\section{Epidemiology}

This domain includes demographics, vital statistics and burden of disease. We are mindful of the quality and recognise the incentives in operations concerning data collection and analysis even for apparently well-run endeavours such as the Demographic Health Survey (DHS) and the like $[23,24]$. 


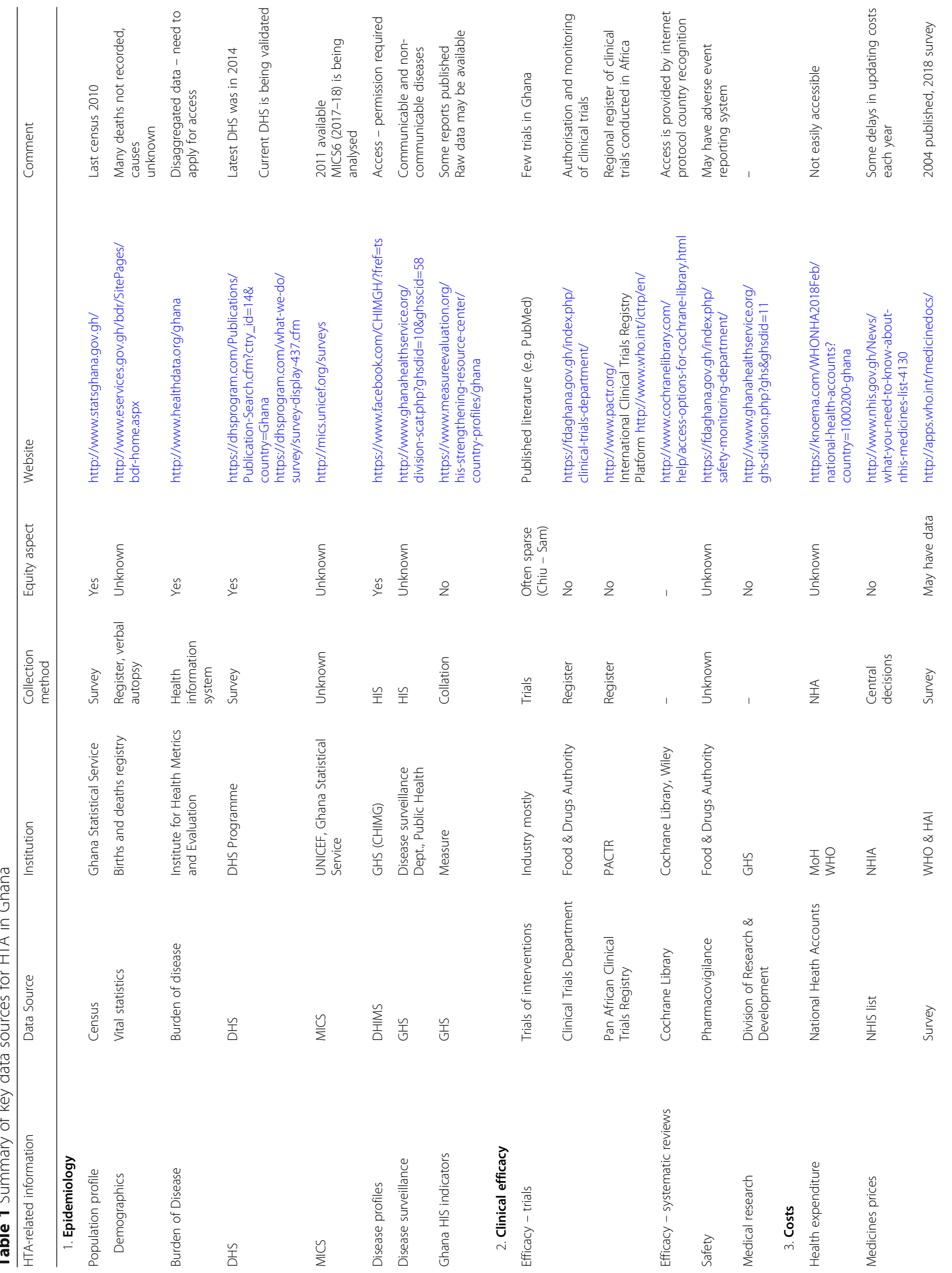




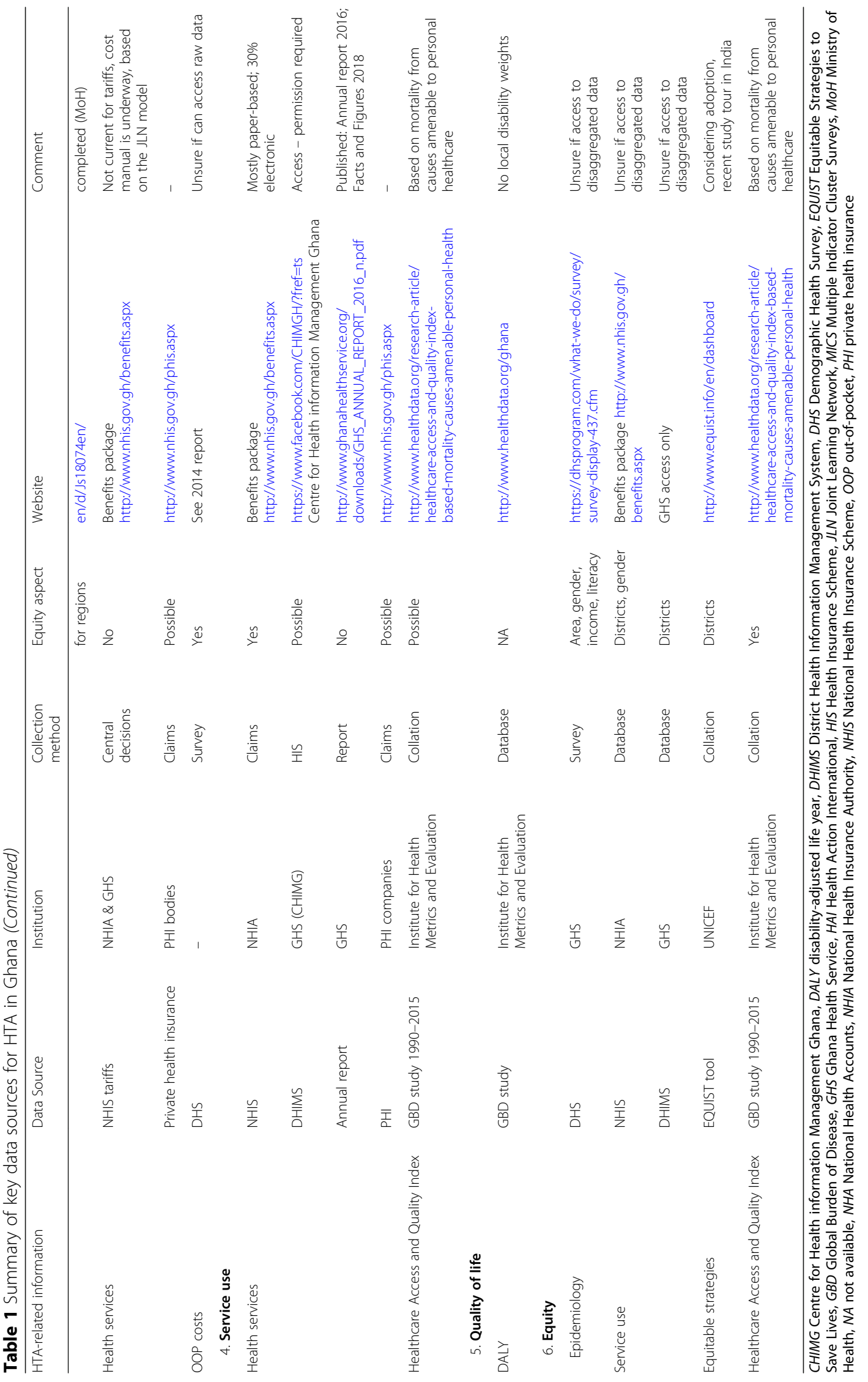




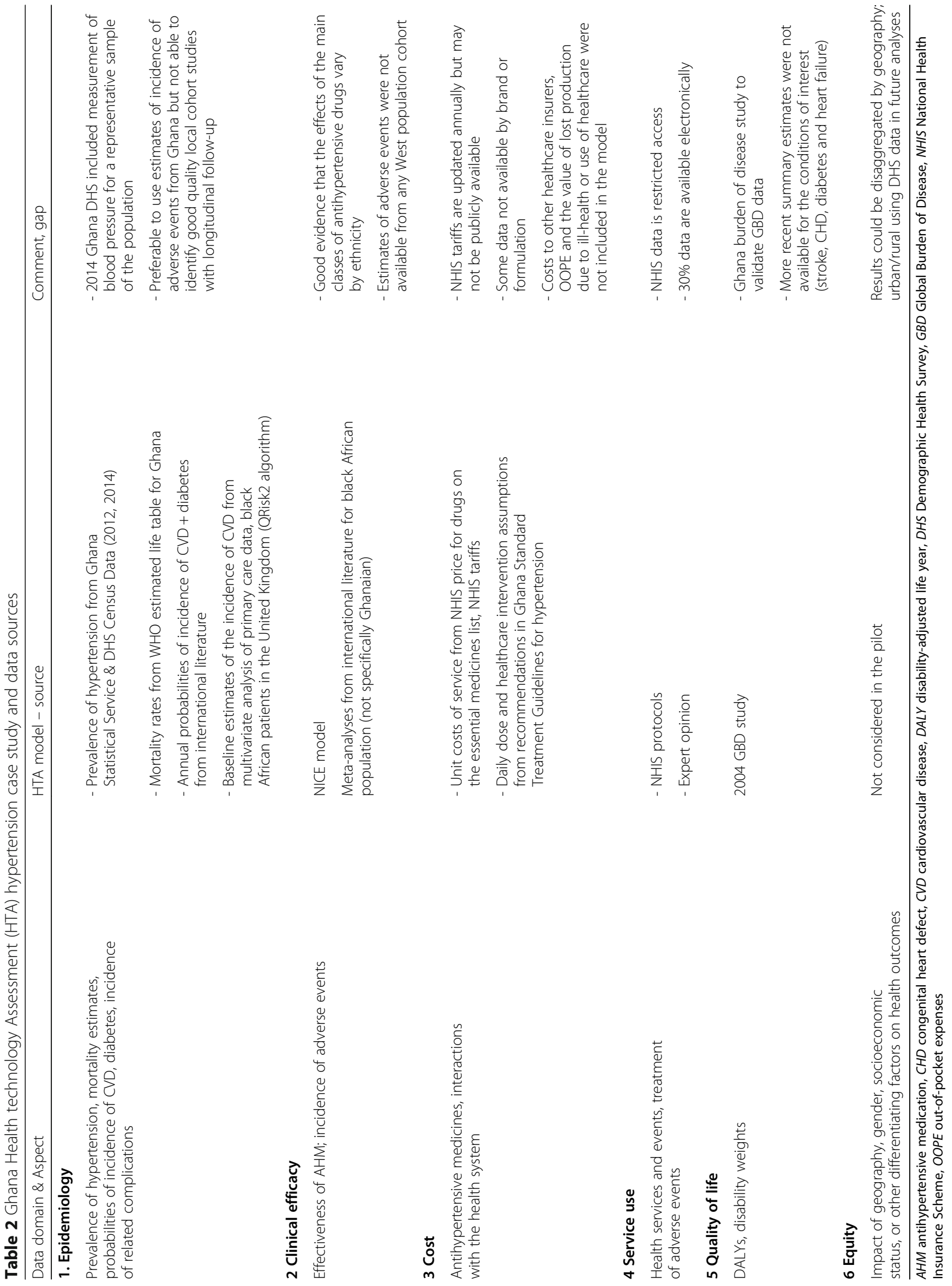




\section{Births and deaths}

The Ghana Statistical Service (GSS) administers the Civil Registration and Vital Statistics system in Ghana [25]. The Civil Registration and Vital Statistics system is a focus of the Bloomberg Philanthropies Data for Health Initiative (https://mspgh.unimelb.edu.au/dataforhealth), which aims to support country systems for counting births, deaths and causes of death in order to better inform health policy; Ghana is a participating country. The GSS can provide the population profile of the country via the census (most recent was in 2010) [26].

\section{Disease burden}

The DHS surveys are conducted every 5 years and are available from 1988; the latest DHS was conducted in 2014 and published in 2015 [27]. The DHS is financially supported by both the Government of Ghana and USAID. The reports and data are freely available but access to more detailed (raw) data can be requested from the GSS. The GSS has been conducting the Ghana Living Standards Survey (GLSS) since 1987/8 (with the latest round being in 2017) with the aim of measuring the living conditions and well-being of the population [28]. The WHO Study on Ageing and Adult Health (SAGE) has disease burden data for Ghana for people aged 50 and above across four waves between 2002 and 2018 [29].

Information on the prevalence and incidence of a disease or condition is necessary when developing economic evaluations and related budget impact analyses, i.e. the latter forecasts expenditure, and possibly savings, usually over a period of 3-5 years. The prevalence data for any common condition (such as hypertension) is likely to be relatively straightforward to obtain using cross-sectional studies in the published literature. However, sourcing data on the incidence of disease (i.e. the occurrence of new cases in the population) is often more challenging. Incidence data are generally obtained from population-based cohort studies where participants are followed over long periods of time. These studies are necessarily expensive to establish and maintain. One potential source of incidence data is from the Ghana Health and Demographic Surveillance Systems (HDSS) that are part of the global International Network for the Demographic Evaluation of Populations and Their Health in Developing Countries (INDEPTH, www. indepth-network.org). The Ghana HDSS centres are a collaboration between the MoH, Ghana Health Service and Ghana Statistical Service. There are three surveillance centres in Ghana - Kintampo (Bono East region), Dodowa (Greater Accra region) and Navrongo (Upper East region). These centres have been following a defined geographical population for many years, so there is large scope for the centres to provide valuable data on the incidence on disease, especially non-communicable diseases (NCD), which are lacking in Ghana. The HDSS sites conduct surveys of individuals and, more recently, have linked these survey data to the district health information management system (DHMIS). Research partners, often from the United Kingdom or countries in Europe, regularly fund specific projects using the HDSS sites. There are clear policies and protocols for data access [30].

Ghana has a number of national control programmes such as those for HIV, malaria and tuberculosis [31]. These programmes calculate incidence rates by using epidemiological modelling using a variety of inputs, including reported new cases. The HIV programme also conducts sentinel surveys. The raw data for facilities is contained in the DHIMS overseen by the Centre for Health Information Management (CHIM; https://www. chimgh.org/) - MoH and Ghana Health Service. The situation of children and women are captured through the Multiple Indicator Cluster Surveys (MICS) from UNICEF and GSS; the MICS6 (2017-18) is currently being analysed but the 2011 data are available [32].

The Institute of Health Metrics and Evaluation (IHME) oversees the Disease Control Priorities Network, which strives to generate reliable, timely and comparable data on the costs and consequences of a broad range of policy options (http://www.healthdata.org/). Ghana is one of eight Disease Control Priorities Network countries, and one of four in sub-Saharan Africa (SSA). The IHME has established the Global Heath Exchange (GHDx) with a comprehensive catalogue of surveys, censuses, vital statistics and other health-related data [33]. Ghana conducted a Burden of Disease study and validated the IHME estimates based on primary data sets and stakeholder consultations with country experts on the various IHME estimates. Some discrepancies were revealed, which were subsequently updated and referred to IHME. These revised burden of disease results were vital to updates of the National Health Policy [34], the Health Sector Medium Term Development Plan [35], and the UHC roadmap for Ghana, which is still being developed (expected December 2019) [36].

\section{Clinical efficacy \\ Clinical trials}

The major sources for clinical efficacy estimates are usually available from randomised controlled trials as published in peer-reviewed health journals and trial repositories (e.g. clinicaltrials.gov). Such trials are often conducted in developed countries with a predominantly white population, raising questions concerning the generalisability of any findings to different ethnic groups. For example, a systematic review showed that hypertensive patients of African ancestry respond better to calcium blockers and diuretics than to ACE inhibitors and 
$\beta$-adrenergic blockers [37]. Clinical trials in NCDs such as cardiovascular diseases and mental health disorders have been virtually non-existent in Ghana [38]. Complex clinical trials of global importance have been conducted in Ghana (e.g. bed nets, rotavirus vaccine, zinc supplements, etc.) and continue to be implemented, mainly by academic and research institutions. The challenge is that there is no central repository to access data from these trials. Evidence from clinical trials, particularly those with more complex interventions in high-income country settings, needs to be considered within the context of implementing such interventions within the Ghanaian health system. Some potential sources of more relevant clinical trials can be found at the clinical trials department of the Ghana Food and Drugs Authority (fdaghana.gov.gh). The clinical trials department authorises (by legal instrument) and monitors clinical trials in Ghana. The Pan African Clinical Trials Registry is a regional register of trials (pactr.org). The Ghana Food and Drugs Authority can also provide information on pharmacovigilance and the safety of medicines.

The recent HTA case study in Ghana for hypertension adapted the United Kingdom National Institute for Health and Care Excellence (NICE) hypertension model [14]. This work aligns with the World Health Assembly resolution of knowledge brokering and use of best practice [39]. Some are currently exploring the issue of adapting existing trials evidence and economic evaluations to suit LMIC settings $[40,41]$.

\section{Systematic reviews and Cochrane Library}

Systematic reviews, especially those from the Cochrane Collaboration, are considered the best available evidence for clinical efficacy. The Cochrane publishers (Wiley) provide free access to the Cochrane Library in over 100 countries, including Ghana (www.cochranelibrary.com/). Access is provided by internet protocol recognition removing the requirement for individual login information.

\section{Costs}

Data on the unit costs of health services, interventions and medicines are crucial for economic evaluations and HTA [22]. When HTA has been used to inform decision-making in countries around the world, the costs used in any economic evaluation commonly tend to focus on resources available to a third-party payer. In Ghana, this would reflect the perspective of the National Health Insurance Authority. In our economic evaluation of antihypertensive medicines, the costs for medicines on the essential medicines list [17] and medical services were derived from the NHIS tariff (available on request). The NHIS tariffs are sometimes dated and a cost manual for services is being developed based on the Joint Learning Network toolkit [42]. The medicines tariffs are revised each year and are derived through a combination of surveys, manufacturer's reference pricing, mark-ups and other considerations. It is a joint process involving the $\mathrm{MoH}$, the Ghana National Drugs Programme, and provider groups. The WHO and Health Action International, in partnership with the $\mathrm{MoH}$, coordinate occasional surveys with a focus on medicines' accessibility and affordability [43]. The most recent survey was published in 2004 [44] but a survey was conducted with $\mathrm{MoH}$ and WHO collaboration in 2018.

In some cases, researchers may be interested in the perspective of the consumer, especially given that only four in ten people are covered by NHIS [12]. Other consumers, particularly if they are not covered by any private health insurance, may be liable for extensive out-of-pocket (OOP) payments [45-47]. The Demographic and Health Survey provides some information on OOP spending [27]. OOP payments are generally estimated using the National Health Accounts [48], the WHO Global Health Expenditures Database [49] and Government budgets. The GLSS also includes some relevant items [28]. The HDSS centre in Navrongo, together with two centres in Burkina Faso and Vietnam, aims to estimate OOP expenditure in a household survey and develop a set of questions to facilitate OOP disease-specific measurement [50]. The $\mathrm{MoH}$ collects information on health expenditure using the $\mathrm{Na}$ tional Health Accounts from the World Bank and OECD and plans to supplement this with local data sources in 2019. They will liaise with the Finance Directorates of all agencies under the $\mathrm{MoH}$ and likely upload it to the upcoming National Health Observatory [51].

\section{Health services use}

Information on the consumption of health services is vital for economic evaluations. For example, when considering an intervention to reduce a cardiovascular event such as a myocardial infarction, it is important to understand the usual treatment pathway for someone who experiences a myocardial infarction and the resulting resource use and costs. If the intervention also reduces the occurrence of stroke, then it is necessary to account for the pathways and resource implications of someone having a stroke. The main source of routinely collected health data is from largely public (and some private) service providers such as NHIS claims and the DHIMS 2. NHIS claims are recorded - the current proportion of electronic claims is 25\% (4th quarter 2019; personal communication, Dsane-Selby) - the rest are paper based [52]. There are annual NHIS reports but there is some delay in making these available online [12]. The NHIS claims data would be an obvious source to explore patterns of use of health services not only at the aggregated level but also, ideally, at the individual level. The DHIMS 2 also has a vast amount of data on health services use [53]. 
Information on pathways of care, particularly for NCDs, is sparse. There are normative standard treatment guidelines [18] but, in the absence of any monitoring data, it is not known to what extent these are implemented in actual practice. Ghana has an essential medicines list $[17,51]$ that guides the medicines available for subsidy on the NHIS [54]. The $\mathrm{MoH}$ is working with the private sector to integrate their service data into the DHIMS. The private health insurance industry in Ghana has 12 providers and mainly covers corporate entities and less-vulnerable populations [55]. There are claims data held by the private health insurance providers, some of which has been made available for research (personal communication, Hollingworth). These data can be used to examine patterns of health service use and costs.

\section{Quality of life}

Health outcomes are often expressed in natural units such as lives saved or a change in some clinical measure such measurement of blood pressure. Economic evaluations that only consider such measures are limited in their usefulness - the relative value of the intervention can only be ascertained within the scope of that health outcome [22]. A more useful measure is one that includes both mortality and morbidity aspects such as disability-adjusted life years (DALY) or quality-adjusted life years (QALY). Each measure requires a valuation of health states (or disability) where one is perfect health and zero is death. Disabilityadjusted life years are widely used, especially in LMIC settings, and are based on the Global Burden of Disease (GBD) study linked to years of living with a disability [56].

An alternative generic measure available to researchers and decision-makers in Ghana is the QALY, often used in high-income settings but relatively uncommon in LMICs, particularly SSA [56]. Estimating QALYs relies on the use of a generic or disease-specific multi-attribute instrument to measure health-related quality of life (HRQoL). The most widely used generic measure of this type is the so-called EQ-5D (the descriptive system comprises of five dimensions) developed by the EuroQol Group [57]. Typically, the measurement of changes in HRQoL are reported directly from patients, but the utility (valuation) of these changes are based on public preferences using a choice-based method (commonly time trade-off in the case of EQ-5D). The valuation exercise is usually undertaken separately and the results are often referred to as the population tariff. However, there are no published EQ-5D (three or five level) value sets for Ghana. An orthopaedic hospital in Accra is administering a multi-attribute instrument (SF-36D) to patients before and after surgery [58]. In Ghana, some authors have evaluated HRQoL in studies encompassing university students' wellbeing (shorter version of the WHO quality of life (WHOQOL-BREF) across four domains) [59], stroke (HRQOLISP-40 instrument across seven domains) [60], asthma (Asthma Quality of Life Questionnaire) [61], and leprosy (disease-specific) short form 20 health survey (six domains) [62]. One study (conference presentation) used the three-level version of EQ-5D in people living with HIV [63]. There is work underway to develop EQ-5D value sets within SSA; sets are now available for Zimbabwe [64] and Ethiopia [65].

\section{Equity}

Again, there are data on equity, depending on the specific aspect and the pragmatic applicability for HTA purposes; DHS, GLSS, and the Study on Ageing and Adult Health (SAGE) have data on equity. Although not necessarily quantified (or quantifiable), HTA processes can explore context-relevant value judgements that may modify interpretation of a cost-effectiveness analysis. Equity considerations are important in priority-setting decisions. These may include aspects relating to equitable access and the provision of health services to vulnerable populations, plus issues relating to gender, socioeconomic indicators, and tribal and religious affiliations. There may also be geographical aspects to consider, including urban versus rural differences in access and outcomes. There may be a pressing need to address inequities in health outcomes for marginalised groups; to do so, data on the relevant characteristics is required.

There are some equity-relevant data available in Ghana. The sample design of the DHS provides estimates at the national and regional levels as well as for urban and rural areas. Wealth quintiles are available for households in the datasets [27]. The GLSS also provides data on wealth quintiles [28] and the census has data for the socioeconomic status at the district level [26]. Equity aspects of service use can be explored through the NHIS and the DHIMS, especially at district and facility level. The three HDSS sites can provide some data; for example, one project aimed to understand the impact of UHC on the poor and vulnerable. The HDSS at Navrongo was one of two impact of UHC study sites (the other in Vietnam) exploring health services need, access, use, quality of care and reliable measurement of socioeconomic status at the district level [66]. The GBD study collaborators published a study on the healthcare access and quality index based on mortality from causes amenable to personal healthcare in 195 countries and territories, including Ghana [67]. The EQUIST tool from UNICEF (http://www.equist.info/en/dashboard) will be linked to the DHMIS (Ghana Health Service) by 2020.

\section{Discussion}

\section{Main findings}

Ghana has several large data sources to support HTA (e.g. DHS, GBD and NHIS) with sufficiently rigorous quality assurance processes. There are few accessible 
data sources for costs and service use. The NHIS claims data are a potentially rich source of data on these but there are access limitations. The initiative to have facilities submit claims through an electronic platform presents an opportunity to harness such a rich source of data for HTA. Data on services and medicines costs are based entirely on NHIS tariffs, but these need to be regularly reviewed and updated. The 2017 value-added tax exemption on medicines should result in an average decrease of $30 \%$ in costs for medicines with the costs of selected medicines decreasing up to 70\% [68]. There are almost no data for the domains of quality of life [69] and equity.

\section{Strengths and limitations}

To the best of the authors' knowledge, this is the first comprehensive study to describe the landscape of health system data sources to inform HTA in Ghana and could serve as a blueprint for other SSA countries [70]. The main limitation was to accurately and comprehensively identify all the sources of data for HTA [21]. We have not been able to consider aspects of quality and missing data. As such, we consider this an important starting point for a more complete description and assessment of data suitable for HTA purposes in Ghana.

WHO urges member states to develop and improve the collection of data as noted in the Health Intervention Technology Assessment Resolution [2]. Much of the data described in this paper are derived from governmentsponsored information systems and census surveys, where statistics are reported to be systematically exaggerated across Africa [24, 71-73]. We did not assess the quality of the data sources in this paper but there are promising quality assurance processes to ensure data integrity for some sources [27, 30, 52]. A more comprehensive consideration of quality could be pursued in future studies.

Measures for safeguarding data should be reviewed and improved across Ghana to ensure robust, highquality evidence capture and protect data against undue political interference [24]. The strength of any economic evaluation lies in the data that populates it, and thus improving the quality of data collected in Ghana, and indeed across Africa, will be a crucial step towards more robust evidence-informed policy decisions. We are currently reviewing the data sources used in selected published economic evaluations from SSA.

\section{Implications for policy and practice}

In terms of epidemiological data, this important component of HTA is adequately served by data available from the GBD and DHS. However, most other contextually relevant data sources are arguably inadequate for the production of analyses that are able to rely on information most relevant to Ghanaian decision problems. Even though the epidemiological data sources are among the most reliable, there remain substantial gaps. There are several studies exploring the prevalence of NCDs, e.g. hypertension in various Ghanaian populations [74-77], but, to our knowledge, there are no studies looking at the incidence of NCDs. This would require a large-scale cohort study and it could consider the main NCDs and comorbidities of interest in Ghana, i.e. cardiovascular disease, type 2 diabetes and cancer, and the associated risk factors including hypertension and obesity. With regard to preference-based health-related quality of life measures, Ghanaian policy-makers will need to be aware of the implications of such data to inform the routine use of QALY measures in HTAs. For example, in the overwhelming majority of countries in SSA, value sets are simply not available for the most commonly, and widely understood, generic HRQoL tool, the EQ-5D (five dimensions). The adoption of QALYs is feasible in SSA [65], and awareness raising together with action and support for research efforts at developing value sets for Ghana through peer or South-South channels may be an important means of methods development [7]. This highlights the importance of having a reference case [22] that signals preference (and creates a demand) for certain types of data [78].

It is important to highlight that, for decision-making purposes, there will always be gaps in the available evidence and, when there is evidence, it may be of varying quality [79]. Analysts and decision-makers will therefore be routinely confronted by uncertainty that needs to be acknowledged and managed. Judgement will be needed in deciding whether the data used in any HTA is the 'best available' for the evaluation problem being examined, and the implications of any bias introduced when using sub-optimal data sources [80]. This further highlights the appropriate conceptualisation of a functioning ('institutionalised') HTA system as one that has both procedural and technical elements [81], is supported politically and has cooperation among key organisations [82]. In relation to the technical elements, a key foundation is establishing a framework (or reference case) for the conduct of economic evaluations in Ghana. Such frameworks not only promote consistency but serve to illuminate the key information required for decisionmaking purposes, and thus help drive more targeted primary research and data collection [22].

At least initially, the new HTA mechanism in Ghana may have to work with less-than-complete access to good quality, contextually relevant data and evidence, and it will likely be necessary to accept some reliance on internationally produced evidence. Adopting approaches that do not apply hierarchies of evidence in an unthinking manner [83] and emphasising 'fitness for purpose' may also assist in helping researchers and decisionmakers in Ghana make the best use of available 
evidence, whether produced domestically or from international sources [84]. For example, if health utilities are required, they could be sourced from the literature and reports from HTA agencies in similar contexts [9]. Likewise, costs should ideally be from Ghanaian sources considering local care pathways but can be supplemented by data from the literature, expert clinical opinion, and costs from similar jurisdictions with appropriate adjustment. As the programme of HTA matures in both the public and private sectors, and especially if its outputs can be used to identify research gaps ideally funded by a dedicated department within the $\mathrm{MoH}$ as a start, and potentially a separate agency in the future, the information sources should improve in quality and constraints should lessen over time. In addition, improving existing information systems (and addressing any governance challenges) should be a priority, not least because such action will have broader health system benefits in enabling better monitoring of performance and supporting links between best practice and remuneration. Indeed, the World Health Assembly resolution on HITA [2] rightly encourages member states to strengthen the routine collection of health system data as a necessary step towards achievement of UHC. The key to HTA is to use the best available data while being open about its limitations and the impact on uncertainty.

\section{Conclusions}

It will be critical that an overarching strategic and mandatory approach to the collection and use of health information is developed for Ghana in parallel to, and informed by, the development of HTA approaches to support resource-allocation decisions. In this context, donors have a critical role in emphasising and supporting the development of health information systems, especially in those settings transitioning from external aid. The latter is particularly important given the track record of aid-funded data collection, which has been mostly about data to ensure progress against globally set targets (Millennium Development Goals, Sustainable Development Goals) as opposed to data needed by countries in order to run their systems to meet these targets [24]. The gathering of quality data to enhance HTA assessments should also be seen as a political choice that the country should champion. This is the only way to achieve UHC in a cost-effective way.

\footnotetext{
Abbreviations

DHIMS: District Health Information Management System; DHS: Demographic Health Survey; EQ-5D : EuroQol five dimensions; GBD: Global Burden of Disease; GLSS: Ghana Living Standards Survey; GSS: Ghana Statistical Service; HDSS: Health and Demographic Surveillance Systems; HRQoL: health-related quality of life; HTA: health technology assessment; IHME: Institute of Health Metrics and Evaluation; LMICs: low- and middle-income countries; MICS: Multiple Indicator Cluster Surveys; $\mathrm{MoH}$ : Ministry of Health; NHIS: National Health Insurance Scheme; NCD: non-communicable diseases; OOP: out-of-pocket; QALYs: quality-adjusted life years; SSA: sub-Saharan Africa; UHC: universal health coverage
}

\section{Acknowledgements}

We thank Ms. Heleen Vellekoop for her contributions on data sources in Ghana. We appreciate the time provided by Dr. Oche and Dr. Kamanda at the INDEPTH secretariat in Accra.

\section{Authors' contributions}

SH developed the project and wrote the first draft. LD, EO, LS, MGL and JN helped define and give details on the data sources in Ghana. LD, FR and KC contributed to the interpretation of the results. All authors read and approved the final manuscript.

\section{Funding}

This work is produced under the international Decision Support Initiative (iDSI). The iDSI is funded by the Bill \& Melinda Gates Foundation (OPP1087363), the UK Department for International Development, and the Rockefeller Foundation. The funders had no role in study design, data collection and analysis, decision to publish, or preparation of the manuscript.

\section{Availability of data and materials}

All data generated or analysed during this study are included in this published article.

Ethics approval and consent to participate

Not applicable.

\section{Consent for publication}

Not applicable.

\section{Competing interests}

The authors declare that they have no competing interests.

\section{Author details}

${ }^{1}$ School of Pharmacy, University of Queensland, 20 Cornwall St, Woolloongabba, QLD 4102, Australia. ${ }^{2}$ School of Public Health, Imperial College London, London, United Kingdom. ${ }^{3}$ iDSI, Imperial College London, London, United Kingdom. ${ }^{4}$ Policy Planning, Monitoring and Evaluation, Ministry of Health, Accra, Ghana. ${ }^{5}$ National Health Insurance Authority, Accra, Ghana. ${ }^{6}$ Ministry of Health, Accra, Ghana. ${ }^{7}$ School of Public Health, University of Ghana, Accra, Ghana. ${ }^{8}$ Center for Global Development, London, United Kingdom.

Received: 19 November 2019 Accepted: 17 March 2020

Published online: 28 April 2020

\section{References}

1. Chalkidou K, Glassman A, Marten R, Vega J, Teerawattananon Y, Tritasavit N, Gyansa-Lutterodt M, Seiter A, Kieny MP, Hofman K, Culyer AJ. Priority-setting for achieving universal health coverage. Bull World Health Organ. 2016;94:462-7.

2. World Health Assembly: WHA67.23 - Health Intervention and Technology Assessment in Support of Universal Health Coverage. WHA Resolution; Sixtyseventh World Health Assembly, 2014. 2014, http://apps.who.int/ medicinedocs/en/m/abstract/Js21463en/: (Accessed 11 November 2019).

3. Sorenson C, Chalkidou K. Reflections on the evolution of health technology assessment in Europe. Health Econ Policy Law. 2012;7:25-45.

4. Chaikledkaew U, Lertpitakpong C, Teerawattananon Y, Thavorncharoensap M, Tangcharoensathien $\mathrm{V}$. The current capacity and future development of economic evaluation for policy decision-making: a survey among researchers and decision-makers in Thailand. Value Health. 2009;12(Suppl 3):S31-5.

5. Teerawattananon Y, Tantivess S, Yothasamut J, Kingkaew P, Chaisiri K. Historical development of health technology assessment in Thailand. Int J Technol Assess Health Care. 2009;25(Suppl 1):241-52.

6. Hofman KJ, McGee S, Chalkidou K, Tantivess S, Culyer AJ. National Health Insurance in South Africa: relevance of a national priority-setting agency. $S$ Afr Med J. 2015;105:739-40.

7. MacQuilkan K, Baker P, Downey L, Ruiz F, Chalkidou K, Prinja S, Zhao K Wilkinson T, Glassman A, Hofman K. Strengthening health technology assessment systems in the global south: a comparative analysis of the HTA journeys of China, India and South Africa. Glob Health Action. 2018;11:1527556.

8. Fasseeh AN, George M, El Rabbat M, Al Rabayah AA, Karam R, Kristensen FB, Moneim ARIA, Jameleddine M, Al-Saggabi AH, Alowayesh MS, et al. HTA 
implementation in Middle East and North Africa: comparison of current and preferred status (PNS179). Value Health. 2019;22:S315-6.

9. Jameleddine M, Grati H, Jebali MC, Kouki M, Gutierrez-Ibarluzea I, Toumi M, Zghal K. Trastuzumab in the treatment of HER2-positive early and locally advanced breast cancer: the first HTA report of INEAS-Tunisia (PCN382). Value in Health. 2019;22:S511.

10. World Health Organization: Using Health Technology Assessment for Universal Health Coverage and Reimbursement Systems. 2016, http://apps. who.int/medicinedocs/en/m/abstract/Js22250en/. Accessed 11 Nov 2019.

11. Wang H, Otoo N, Dsane-Selby L. Ghana National Health Insurance Scheme: Improving Financial Sustainability Based on Expenditure Review. In: World Bank Studies. Washington, DC: World Bank; 2017.

12. National Health Insurance Authority: National Health Insurance Scheme Annual Reports. 2019. http://www.nhis.gov.gh/annualreports.aspx. Accessed 11 Nov 2019.

13. Merriman B, Wilkerson T, Heath M, Kelly B, Eghan K, Ghana Health Insurance Analysis 2016. Submitted to the US Agency for International Development by the Systems for Improved Access to Pharmaceuticals and Services (SIAPS) Program Arlington. VA: Management Sciences for Health; 2016

14. Gad M, Lord J, Chalkidou K, Gyansa-Lutterodt M, Asare B, Ruiz F. Supporting the development of evidence-informed policy options: an economic evaluation of hypertension management in Ghana. Value Health. 2020;23:171-9.

15. Hollingworth S, Gyansa-Lutterodt M, Dsane-Selby L, Nonvignon J, Lopert R, Gad M, Ruiz F, Tunis S, Chalkidou K. Implementing health technology assessment in Ghana to support UHC: building relationships that focus on people, policy and process. Int J Technol Assess Health Care. 2020;36:8-11.

16. Ministry of Health Republic of Ghana. National Medicines Policy. Accra: $\mathrm{MoH} ; 2018$.

17. Ministry of Health Republic of Ghana. Essential Medicines List. Seventh Edition. Accra: MoH; 2017.

18. Ministry of Health Republic of Ghana. Standard Treatment Guidelines. Seventh Edition. Accra: MoH; 2017.

19. Ghana Statistical Service. Ghana's Data for Sustainable Development Roadmap Forum. Summary Report 5-6 April 2017. Accra: GSS; 2017.

20. Chalkidou K, Marquez P, Dhillon PK, Teerawattananon Y, Anothaisintawee T, Gadelha CA, Sullivan R. Evidence-informed frameworks for cost-effective cancer care and prevention in low, middle, and high-income countries. Lancet Oncol. 2014;15:e119-31.

21. Downey LE, Rao N, Guiness L, Asaria M, Prinja S, Sinha P, Kant R, Pandey A Cluzeau F, Chalkidou K. Identification of publicly available data sources to inform the conduct of Health Technology Assessment in India [version 2; peer review: 3 approved]. F1000Res. 2018;7:245.

22. Wilkinson T, Sculpher MJ, Claxton K, Revill P, Briggs A, Cairns JA, Teerawattananon Y, Asfaw E, Lopert R, Culyer AJ, Walker DG. The International Decision Support Initiative Reference Case for Economic Evaluation: An Aid to Thought. Value Health. 2016;19:921-8.

23. Rajan SI, James KS. Third national family health survey in India: issues, problems and prospects. Econ Polit Weekly. 2008:43:33-8.

24. Sandefur J, Glassman A. The political economy of bad data: evidence from African Survey and Administrative Statistics. J Dev Stud. 2015;51:116-32.

25. Ghana Statistical Service: Civil Registration and Vital Statistics System in Ghana. A Comprehensive Assessment. 2015. https://www.unicef.org/ghana/reports/ civil-registration-and-vital-statistics-system-ghana-0. Accessed 11 Nov 2019.

26. Ghana Statistical Service: Population projection. 2019. http://www. statsghana.gov.gh/nationalaccount_macros.php? Stats= MTA1NTY1NjgxLjUwNg==/webstats/s679n2sn87. Accessed 11 Nov 2019.

27. Ghana Statistical Service (GSS), Ghana Health Service (GHS), ICF International. 2014 Ghana Demographic and Health Survey (DHS) Key Findings. Rockville: GSS, GHS, and ICF International; 2015.

28. Ghana Statistical Service: Ghana - Ghana Living Standard Survey (GLSS 7) 2017. 2017. http://www2.statsghana.gov.gh/nada/index.php/catalog/97/ study-description. Accessed 11 Nov 2019.

29. World Health Organization: Study on Global AGEing and Adult Health (SAGE) 2020. https:/www.who.int/healthinfo/sage/cohorts/en/. Accessed 11 Nov 2019.

30. INDEPTH Network: Data Sharing and Access Policies and Protocols. 2019 http://www.indepth-network.org/data-stats/data-sharing-and-access-policiesand-protocols. Accessed 11 Nov 2019.

31. Ghana Health Service: Ghana Health Service Health Programmes. 2018. http:// www.ghanahealthservice.org/programme-list.php. Accessed 11 Nov 2019.

32. Ghana Statistical Service: Ghana Multiple Indicator Cluster Survey with an Enhanced Malaria Module and Biomarker, 2011, Final Report. https://www. unicef.org/ghana/Ghana_MICS_Final.pdf. Accessed 11 Nov 2019.
33. Institute of Health Metrics and Evaluation: Global Health Data Exchange Ghana. 2019. http://ghdx.healthdata.org/geography/ghana. Accessed 11 Nov 2019.

34. Ministry of Health Republic of Ghana: National Health Policy. Creating Wealth Through Health. 2007. http://www.moh.gov.gh/wp-content/ uploads/2016/02/national-health-policy.pdf. Accessed 11 Nov 2019.

35. Ministry of Health Republic of Ghana: The Health Sector Medium Term Development Plan 2014-2017. 2014. Accessed 14 Nov 2018.

36. News Desk. MoH Outdoors UHC Roadmap. Modern Ghana. 2019; https:// www.modernghana.com/news/925792/moh-outdoors-uhc-roadmap.html. Accessed 11 Nov 2019.

37. Brewster $L M$, Seedat $Y K$. Why do hypertensive patients of African ancestry respond better to calcium blockers and diuretics than to ACE inhibitors and beta-adrenergic blockers? A systematic review. BMC Med. 2013;11:141.

38. Adjei GO, Sulley AM. Clinical trials in Ghana: evolution and current landscape. Int J Clin Trials. 2014;1:78-86.

39. World Health Assembly: Ministerial Summit on Health Research (WHA58.34). 2005. https:/apps.who.int/iris/handle/10665/20384. Accessed 23 May 2019.

40. Alshreef A, Macquilkan K, Dawkins B, Riddin J, Ward S, Meads D, Taylor M, Dixon S, Culyer T, Hofman K, et al. Assessing the appropriateness of existing model adaptation methods for low and middle-income countries. Value Health. 2018;21:S8

41. Mealing S. Assessing the Appropriateness of Existing Model Adaptation Methods for Low and Middle Income Countries (LMICS). Tokyo: ISPOR Asia Pacific Conference; 2018. https://www.sheffield.ac.uk/polopoly_fs/1.804454 !/file/ISPOR_AP_Tokyo_Model_Adaptation.pdf. Accessed 11 Nov 2019.

42. Joint Learning Network Costing Collaborative: Costing of Health Services for Provider Payment: A Practical Manual. 2019. http://www. jointlearningnetwork.org/resources/costing-of-health-services-for-providerpayment-a-practical-manual. Accessed 11 Nov 2019.

43. Ministry of Health Republic of Ghana: Medicine Prices in Ghana: A Comparative Study of Public, Private and Mission Sector Medicine Prices (WHO \& HAl). 2004. http://apps.who.int/medicinedocs/en/d/Js18074en/. Accessed 11 Nov 2019.

44. Ministry of Health Republic of Ghana: Medicine Prices in Ghana (WHO \& HAI). 2004. Accessed 7 Jun 2019.

45. Akazili J, Gyapong J, McIntyre D. Who pays for health care in Ghana? Int J Equity Health. 2011;10:26.

46. Akazili J, McIntyre D, Kanmiki EW, Gyapong J, Oduro A, Sankoh O, Ataguba JE. Assessing the catastrophic effects of out-of-pocket healthcare payments prior to the uptake of a nationwide health insurance scheme in Ghana. Glob Health Action. 2017;10:1289735.

47. Aryeetey GC, Westeneng J, Spaan E, Jehu-Appiah C, Agyepong IA, Baltussen R. Can health insurance protect against out-of-pocket and catastrophic expenditures and also support poverty reduction? Evidence from Ghana's National Health Insurance Scheme. Int J Equity Health. 2016;15:116.

48. Ministry of Health Republic of Ghana: Ghana National Health Accounts 2005 \& 2010. 2013. http://www.moh.gov.gh/wp-content/uploads/2016/02/GhanaNational-Health-Accounts-2005-2010.pdf. Accessed 11 Nov 2019.

49. World Health Organization: Global Health Expenditure Database. 2019. https://apps.who.int/nha/database. Accessed 11 Nov 2019.

50. INDEPTH Network: Out of Pocket Spending (iHOPE). 2019. http://www. indepth-network.org/projects/ihope. Accessed 11 Nov 2019.

51. World Health Organization: Global Health Observatory (GHO) Data - Ghana. 2019. https://www.who.int/gho/countries/gha/en/. Accessed 14 Nov 2019.

52. Nsiah-Boateng E, Asenso-Boadi F, Dsane-Selby L, Andoh-Adjei FX, Otoo N, Akweongo P, Aikins M. Reducing medical claims cost to Ghana's National Health Insurance scheme: a cross-sectional comparative assessment of the paper- and electronic-based claims reviews. BMC Health Serv Res. 2017;17:115.

53. Adaletey DL, Poppe O, Braa J. Cloud Computing for Development Improving the Health Information System in Ghana. IST-Africa 2013 Conference Proceedings Paul Cunningham and Miriam Cunningham (Eds) IIMC International Information Management Corporation. Oslo: University of Oslo; 2013. https://www.duo.uio.no/handle/10852/37209. Accessed 15 Apr 2020

54. National Health Insurance Authority: National Health Insurance Scheme Medicines List. 2019. http://www.nhis.gov.gh/medlist.aspx. Accessed 11 Nov 2019.

55. Ampah N, Chalkidou K, Hollingworth S. The private health insurance sector (PHIS) in Ghana: what is their role in the path to universal health coverage (UHC)? African Health Economics and Policy Association 5th Biennial Conference, 2019, 11-14 March 2019. Accra. 
56. Leech AA, Kim DD, Cohen JT, Neumann PJ. Use and misuse of costeffectiveness analysis thresholds in low- and middle-income countries: trends in cost-per-DALY studies. Value Health. 2018;21:759-61.

57. EuroQol Office: EQ-5D-3L Population Norms. 2019. https://euroqol.org/eq-5 d-instruments/eq-5d-3l-about/population-norms/. Accessed 11 Nov 2019.

58. Cooper V, Clatworthy J, Harding R, Whetham J, Emerge C. Measuring quality of life among people living with HIV: a systematic review of reviews. Health Qual Life Outcomes. 2017;15:220.

59. Opoku-Acheampong A, Kretchy IA, Acheampong F, Afrane BA, Ashong S, Tamakloe B, Nyarko AK. Perceived stress and quality of life of pharmacy students in University of Ghana. BMC Res Notes. 2017;10:115.

60. Donkor ES, Owolabi MO, Bampoh PO, Amoo PK, Aspelund T, Gudnason V. Profile and health-related quality of life of Ghanaian stroke survivors. Clin Interv Aging. 2014;9:1701-8.

61. Anum PO, Anto BP, Forson AG. Structured pharmaceutical care improves the health-related quality of life of patients with asthma. J Pharmaceut Policy Pract. 2017;10:8

62. Bello Al, Dengzee SA, lyor FT. Health related quality of life amongst people affected by leprosy in South Ghana: a needs assessment. Lepr Rev. 2013;84: 76-84.

63. Sackey J, Zhang FF, Rogers B, Aryeetey R, Wanke C. Impact on depression and health-related quality of life among people living with HIV in Accra, Ghana (Abstract 312.2). FASEB J. 2017:31:312 2.

64. Szende A, Janssen B, Cabases J. Self-Reported Population Health: An International Perspective Based on EQ-5D. Dordrecht: Springer; 2014

65. Welie AG, Gebretekle GB, Stolk E, Mukuria C, Krahn MD, Enquoselassie F, Fenta TG. Valuing health state: an EQ-5D-5L value set for Ethiopians. Value Health Reg Issues. 2019;22:7-14.

66. INDEPTH Network: Impact of Universal Health Coverage (IUHC). 2018. http:// www.indepth-network.org/projects/iuhc. Accessed 11 Nov 2019.

67. GBD 2015 Healthcare Access and Quality Collaborators. Healthcare Access and Quality Index based on mortality from causes amenable to personal health care in 195 countries and territories, 1990-2015: a novel analysis from the Global Burden of Disease Study 2015. Lancet. 2017:390:231-66.

68. Government of Ghana. Value Added Tax (VAT) Exemption Legislative Instrument (LI) 2255 of Nov 2017. Accra: Ghana Assembly Press; 2017.

69. Kularatna S, Whitty JA, Johnson NW, Scuffham PA. Health state valuation in low- and middle-income countries: a systematic review of the literature. Value Health. 2013;16:1091-9.

70. Data Use Partnership \& PATH: The journey to better data for better health in Tanzania 2017-2023. 2016. http://www.path.org/publications/detail. php?i=2734. Accessed 11 Nov 2019.

71. Glassman A, Ezeh A, McQueston K, Brinton J, Ottenhoff J: Delivering on the Data Revolution in Sub-Saharan Africa. Final Report of the Data for African Development Group. A Report from the Centre for Global Development and The African Population and Health Research Center. 2014. https://www. cgdev.org/publication/delivering-data-revolution-sub-saharan-africa-0. Accessed 11 Nov 2019.

72. Jerven $M$, Johnston D. Statistical Tragedy in Africa? Evaluating the data base for African economic development. J Dev Stud. 2015;51:111-5.

73. Kinyondo A, Pelizzo R. Poor quality of data in Africa: what are the issues? Politics Policy. 2018;46:851-77.

74. Agyemang C, Bruijnzeels MA, Owusu-Dabo E. Factors associated with hypertension awareness, treatment, and control in Ghana, West Africa. J Hum Hypertens. 2006;20:67-71.

75. Agyemang C, Nyaaba G, Beune E, Meeks K, Owusu-Dabo E, Addo J, Aikins AD, Mockenhaupt FP, Bahendeka S, Danquah I, et al. Variations in hypertension awareness, treatment, and control among Ghanaian migrants living in Amsterdam, Berlin, London, and nonmigrant Ghanaians living in rural and urban Ghana - the RODAM study. J Hypertens. 2018;36:169-77.

76. Atinyi R, Takramah W, Axame WK, Owusu R, Parbey PA, Takase M, Adjuik M, Tarkang E, Kweku M. Prevalence and awareness of Hypertension among urban and rural Adults in the Keta Municipality, Ghana. J Med Res. 2017;3:155-63.

77. Bosu WK, Aheto JMK, Zucchelli E, Reilly S. Prevalence, awareness, and associated risk factors of hypertension in older adults in Africa: a systematic review and meta-analysis protocol. Syst Rev. 2017;6:192.

78. Brazier J, Ara R, Azzabi I, Busschbach J, Chevrou-Severac H, Crawford B, Cruz L, Karnon J, Lloyd A, Paisley S, Pickard AS. Identification, review, and use of health state utilities in cost-effectiveness models: an ISPOR Good Practices for Outcomes Research Task Force Report. Value Health. 2019;22:267-75.
79. Rodriguez DC, Hoe C, Dale EM, Rahman MH, Akhter S, Hafeez A, Irava W, Rajbangshi P, Roman T, Tirdea M, et al. Assessing the capacity of ministries of health to use research in decision-making: conceptual framework and tool. Health Res Policy Syst. 2017;15:65.

80. Rawlins MD, Culyer AJ. National Institute for Clinical Excellence and its value judgments. BMJ. 2004;329:224-7.

81. Kriza C, Hanass-Hancock J, Odame EA, Deghaye N, Aman R, Wahlster P, Marin M, Gebe N, Akhwale W, Wachsmuth I, Kolominsky-Rabas PL. A systematic review of health technology assessment tools in sub-Saharan Africa: methodological issues and implications. Health Res Policy Syst. 2014; $12: 66$

82. Li R, Ruiz F, Culyer AJ, Chalkidou K, Hofman KJ. Evidence-informed capacity building for setting health priorities in low- and middle-income countries: a framework and recommendations for further research [version 1; peer review: 2 approved]. F1000Res. 2017:6:231 https://doi.org/210.12688/f11 000research.10966.12681.

83. Rawlins M. De Testimonio: on the evidence for decisions about the use of therapeutic interventions. Clin Med. 2008;8:579-88.

84. Albert MA, Fretheim A, Maiga D. Factors influencing the utilization of research findings by health policy-makers in a developing country: the selection of Mali's essential medicines. Health Res Policy Syst. 2007;5:2.

\section{Publisher's Note}

Springer Nature remains neutral with regard to jurisdictional claims in published maps and institutional affiliations.

Ready to submit your research? Choose BMC and benefit from:

- fast, convenient online submission

- thorough peer review by experienced researchers in your field

- rapid publication on acceptance

- support for research data, including large and complex data types

- gold Open Access which fosters wider collaboration and increased citations

- maximum visibility for your research: over $100 \mathrm{M}$ website views per year

At BMC, research is always in progress.

Learn more biomedcentral.com/submissions 